\title{
Evaluation of The Relationship Between Apelin 36 and Oxidative Stress in Patients with General Anxiety Disorder
}

\author{
Ilkay Bahceci ${ }^{1(\mathrm{ID})}$, Umut Serkan Soztanaci ${ }^{2(\mathrm{ID})}$, Meltem Pusuroglu ${ }^{3(\mathrm{ID})}$,Nuray Arslan ${ }^{1(\mathrm{ID})}$, Omer \\ Faruk Duran ${ }^{1(\underline{I D})}$, Bulent Bahceci ${ }^{3(\mathrm{ID})}$, Zihni Acar Yazici ${ }^{1(\mathrm{ID})}$ \\ ${ }^{1}$ Department of Medical Microbiology, Faculty of Medicine, Recep Tayyip Erdoğan University, Rize, Turkey \\ 2 Department of Anatomy, Hamidiye Faculty of Medicine, Health Sciences University, Istanbul,Turkey \\ 3. Psychiatry Department, Medical Faculty Hospital, Recep Tayyip Erdogan University, Rize, Turkey
}

Copyright@ Author(s) - Available online at https://dergipark.org.tr/en/pub/mbsjohs

Content of this journal is licensed under a Creative Commons Attribution-NonCommercial 4.0 International

License,

18 November 2021, Accepted: 13 December 2021, Published online: 31 December 2021

(C) Ordu University Institute of Health Sciences, Turkey, 2021

\begin{abstract}
Objective: The aim of this study was to evaluate the relationship between apelin-36 and oxidative parameters and Generalized Anxiety Disorder (GAD). Apelin which prevents hippocampal neuronal death is an endogenous ligand for $\mathrm{G}$ protein bound APJ receptors in the central nervous system. Oxidative Stress were occurred by free radicals which caused apoptosis in the hypothlamus, hippocampus and amygdala regions of the Central Nervous System (CNS).

Methods: In this study, 61 patients diagnosed generalized anxiety disorder at psychiatry polyclinic and 55 control subjects were enrolled. The Structured Clinical Interview for DSM-IV Axis I Disorders (SCID-I) and the Hamilton Anxiety Rating Scale (HARS) were used in this study and they were filled by the patients and healthy individuals. Serum apelin-36 level was measured by using an ELISA kit. Total antioxidant status (TAS) and Total Oxidant Status (TOS) in the serum was measured by an automated system. The data were analyzed by using the SPSS.

Results: It was found that serum apelin-36, TOS and Oxidative stress index (OSI) levels were significantly lower in patients' group than controls. In addition, negative correlation was detected between apelin 36 levels and HARS scores, TOS and OSI values in patients' group. TAS values were found as similar between the patient and control group. There was no correlation between TAS and Apelin 36 in the patient group.

Conclusion: In this study it was found that serum apelin-36, TOS and OSI levels were low in GAD. According to the results, Apelin-36 and oxidative stress may play a role in the etiopathogenesis of Generalized Anxiety Disorder.

Key words: Apelin-36, Generalized Anxiety Disorder, Oxidative Stress, Total Antioxidant Status, Total Oxidant Status

Suggested Citation: Bahceci I, Soztanaci US, Pusuroglu M, Arslan N, Duran O F, Bahceci B, Yazici ZA. Evaluation of The Relationship Between Apelin 36 and Oxidative Stress in Patients with General Anxiety Disorder. Mid Blac Sea Journal of Health Science, 2021; 7(3):397-403
\end{abstract}

\section{Address for correspondence/reprints:}

Ilkay Bahceci

Telephone number: +90 (464) 2236126

E-mail: mdilkaybahceci@gmail.com

\section{Introduction}

Generalized anxiety disorder (GAD) is a psychiatric disorder that significantly decreased the quality of life. The excessive fear and worry about the different life events which persist throughout the day are the clinical symptoms of the disease which happened on almost every day. Its annual prevalence is $3-8 \%$. GAD usually begins at twenties and $30 \%$ of GAD referrals take place during this period. 
The presence of other comorbid psychiatric diseases such as depression is $81 \%$ of GAD patients may cause misdiagnosing and decrease the rate of GAD diagnosis

The etiopathogenesis of GAD has not been completely understood yet (1). Neurobiological studies have shown that the level of proinflammatory cytokines is positively correlated with anxiety level (2).

It was determined that neuronal death occurred due to apoptosis based on chronic stress as a result of decreased level of anti-apoptotic protein $\mathrm{Bcl}-2$ in neurons (3).

Magnetic resonance imaging studies have also showed pathological changes in brain regions such as amygdala, hippocampus and hypothalamus (4).

Apelin is a peptide that was firstly purified from bovine stomach tissue extracts. It is synthesized primarily as Pre-pro-apelin which is 77 -amino acid in length. Subsequently, it breakdowns into apelin-13, 17 and 36 forms which had similar bioactivity and action as proteolytically. Apelin performs its function in the central nervous system by binding the $G$ protein-coupled APJ receptor $(5,6)$.

In some studies, it has been shown that apelin 36 reduces hippocampal neuronal death due to cerebral edema and infarcts more effectively than apelin-13 (7-10).

The effects of apelin peptides in the cardiovascular and endocrinological diseases in humans and animals have been studied but the roles in psychiatric diseases have been investigated to a limited extent (11-18).

Oxidative stress occurs by the accumulation of free radicals in cells due to imbalance between oxidants and anti-oxidants. Free radicals reduce synaptic plasticity, increase proinflammatory cytokine secretion and induce apoptosis in the hippocampus, amygdala and hypothalamus (19).

There are no studies investigating the contribution of the relationship between apelin-36 and oxidative stress in GAD etiopathogenesis.

The aim of this study is to investigate the role of oxidative stress and apelin-36 in the etiopathogenesis of GAD.

\section{Methods}

\section{Participants:}

This study was carried out in the outpatient psychiatry clinic of Recep Tayyip Erdoğan Education and Research Hospital. The study was approved by the Ethics Committee of the Medical School (Decision No: 2016/96, Date: 21/10/2016). The study included 61 patients, who were admitted to the outpatient clinic, and 55 healthy individuals, who were hospital staffs.

Voluntary informed consent form was signed by the patients and controls.

Structured Clinical Interview for DSM-IV Axis I Disorders (SCID-I), Hamilton Anxiety Rating Scale (HARS) and sociodemographic data forms were filled by all the individuals themselves.

Individuals who were smoking, using alcohol and drug who had chronic diseases and were pregnancy excluded from the study. Their Body Mass Index ranged between 18.5 and 24.9. Individuals have psychiatric diseases in the control group are excluded and there were no psychiatric diseases except the GAD in the patient group.

\section{Blood Samples:}

After overnight fasting, $5 \mathrm{ml}$ peripheral blood was drawn and then was centrifuged at $4000 \mathrm{rpm} / 5 \mathrm{~min}$. and serum was stored-frozen at $-20{ }^{\circ} \mathrm{C}$ until usage. Serum apelin-36 level was measured by using an ELISA kit (AP 36 test kit, Cloud-Clone Corp., Houston, USA). Sensitivity of the test is reported as $<2.52 \mathrm{pg} / \mathrm{mL}$, according to the manufacturer's protocol. The absorbance was determined at $450 \mathrm{~nm}$ with a microtiter plate reader (Multiskan GO, Thermo Scientific, Waltham, MA, USA) within 5 minutes.

Levels of apelin were measured by the standard curve created by Titri ELISA software. The standard curve was then used to convert the absorbance values to apelin-36 concentration.

Measurement of total antioxidant status (TAS):

TAS in the serum was measured by an automated system. The assay measure anti-oxidative effect against free radical in the serum. The results were expressed as mmol Trolox Eq/L (20).

Measurement of total oxidant status (TOS):

Serum TOS was measured by the automated method of Erel. The results are expressed as micromolar hydrogen peroxide equivalent per liter ( $\mu \mathrm{mol} \mathrm{H} 2 \mathrm{O} 2$ Equiv/L) (20).

\section{Oxidative stress index (OSI):}

OSI (Arbitrary unit) $=$ TOS $(\mathrm{mmol} H 2 \mathrm{O} 2$ Equiv.L-1)/ TAS (mmolTroloxEq/L) (20).

\section{Evaluations of Tests:}

The sociodemographic data form designed by the investigators, which was contained information for age, gender, place of residence, marital status, education level and income status (low: $<500$ euro, 
medium:501-1500 euro, high:>1501 euro) were fulfilled by participants.

The Structured Clinical Interview for DSM-IV Axis I Disorders (SCID-I) was developed by First et al. in 1997 (21). The validity and reliability studies for the adaptation of the SCID-I were performed by Corapcioglu et al. in 1999 (22). The Hamilton Anxiety Rating Scale (HARS) was developed by Hamilton in 1959, which contains 14 questions that query mental and physical symptoms (23).

Each item is rated on a five-point Likert-type scale ranging from 0 to 4 . It determines the level of anxiety and symptom distribution in patients. The validity and reliability study of the Turkish version of the scale was conducted by Yazici et al. in 1998 (24).

\section{Statistical analysis}

Statistical data were analyzed by using SPSS version 18.0. (SPSS Inc., Chicago, IL, USA).

Normal distribution of continuous variables was tested with Kolmogorov-Smirnov test.

Between-group comparisons with normal distribution values were tested by the student's-test.

Chi-square test was used for the comparisons of categorical variables. Correlation analysis was performed by using the Pearson correlation coefficient. Data is expressed as mean \pm standard deviation (SD). The significance level was determined as $\mathrm{p}<0.05$.

\section{Results}

Age and gender distribution were found similar between patient and control groups, respectively, in terms of age $(30.86 \pm 7.61$, range $24-49$, and $32.86 \pm 5.25$, range $23-51, \mathrm{p}=0.342)$ and gender (37 $\mathrm{F} / 24 \mathrm{M}$ and $32 \mathrm{~F} / 23 \mathrm{M}, \mathrm{p}=0.232$ ) (Table 1).

Also,distribution of the place of residence, marital status, education level and income status were found similar between the patient and control groups, respectively $(\mathrm{p}=0.543, \mathrm{p}=0.212, \mathrm{p}=0.303, \mathrm{p}=0.123)$ (Table 1).

There was a statistically significant difference between the two groups in terms of the HARS scores. The scores of the patient group were higher than the control group $(23.46 \pm 4.10$ and $3.86 \pm 1.69$, respectively, $(\mathrm{p} \leq 0.01)$ (Table 2$)$.

The serum apelin-36 levels were statistically significantly lower in the patient group than control group. Levels were measured as $363.83 \pm 21.07 \mathrm{pg} / \mathrm{ml}$ and $1050.93 \pm 51.35 \mathrm{pg} / \mathrm{ml}$, respectively, $(\mathrm{p} \leq 0.01)$ (Table 2 and Figure 1).

Table 1. Sociodemographic Data of Patients and Controls

\begin{tabular}{|c|c|c|c|c|}
\hline Parameters & & Patient $(n=61)$ & Control $(n=55)$ & $\mathrm{p}$ \\
\hline Age (range) & & $30.86 \pm 7.61(24-49)$ & $32.86 \pm 5.25(23-51)$ & 0.342 \\
\hline Gender (F/M) & & $37 / 24$ & $32 / 23$ & 0.232 \\
\hline \multirow[t]{3}{*}{ Place of residence } & City & 36 & 32 & \multirow{3}{*}{0.543} \\
\hline & Village & 20 & 17 & \\
\hline & Town & 13 & 6 & \\
\hline \multirow[t]{3}{*}{ Marital status } & Single & 38 & 34 & \multirow{3}{*}{0.212} \\
\hline & Widow(er) & 21 & 19 & \\
\hline & Divorcee & 2 & 2 & \\
\hline \multirow[t]{3}{*}{ Education level } & Primary & 10 & 7 & \multirow{3}{*}{0.303} \\
\hline & Middle & 37 & 35 & \\
\hline & High & 14 & 13 & \\
\hline \multirow[t]{3}{*}{ Income level } & Low & 15 & 13 & \multirow{3}{*}{0.123} \\
\hline & Medium & 38 & 35 & \\
\hline & High & 8 & 7 & \\
\hline
\end{tabular}


Table 2. Parameters measured and outcomes

\begin{tabular}{llll}
\hline & Patient & Control & p \\
\hline HARS* & $23.46 \pm 4.10$ & $3.86 \pm 1.69$ & 0.000 \\
Apelin 36 $(\mathrm{pg} / \mathrm{ml})$ & $363.83 \pm 21.077$ & $1050.93 \pm 51.35$ & 0.000 \\
TAS* $(\mathrm{mmol} \mathrm{Trolox} \mathrm{Eq/L)}$ & $1.39+0.81$ & $1.32+0.20$ & 0.721 \\
TOS* $\left(\mathrm{mmol} \mathrm{H} \mathrm{O}_{2}\right.$ Equiv./L) & $44.52+0.28$ & $10.02+0.97$ & 0.005 \\
OSI* (arbitrary unit) & $3.92+6.17$ & $0.78+0.44$ & 0.005 \\
\hline
\end{tabular}

*Abbreviations: HARS (Hamilton Anxiety Rating Scale), TAS (Total Antioxidant Status), TOS (Total Oxidant Status), OSI (Oxidative Stress Index)

Table 3. The correlation of TAS, TOS, OSI and HARS scores with apelin 36 levels in patients

\section{Apelin 36}

$\begin{array}{lll} & \mathrm{r} & \mathrm{p} \\ \text { HARS* } & -0.737 & 0.000^{*} \\ \text { TAS* }^{*} & -0.126 & 0.233 \\ \text { TOS* }^{*} & -0.443 & 0.026^{*} \\ \text { OSI* }^{*} & -0.374 & 0.043^{*}\end{array}$

Pearson correlation, $\mathrm{p}<0.05$.

*Abbreviations: HARS (Hamilton Anxiety Rating Scale), TAS (Total Antioxidant Status), TOS (Total Oxidant Status), OSI (Oxidative Stress Index)

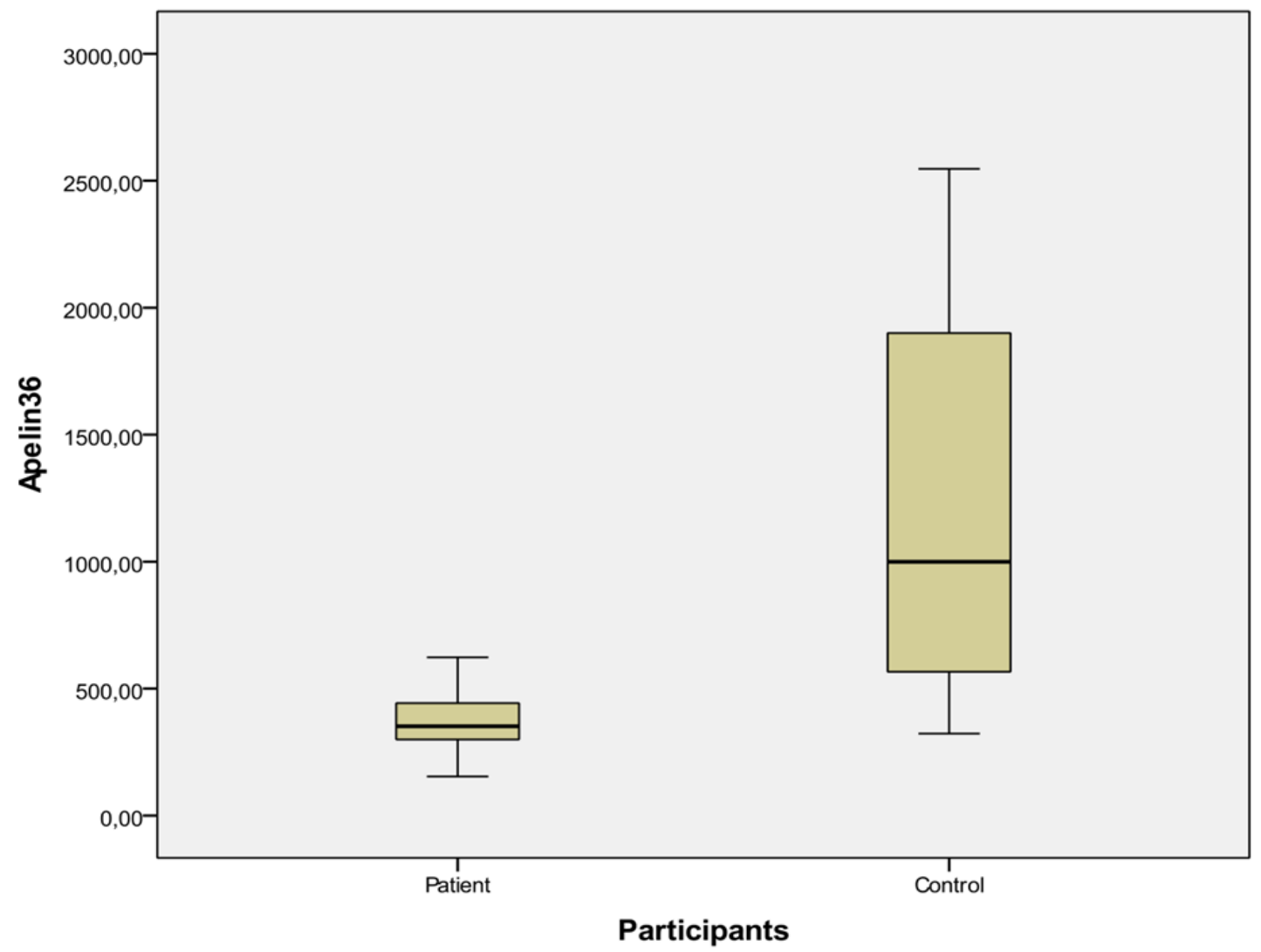

Figure 1. The distribution of apelin-36 levels $(\mathrm{pg} / \mathrm{ml})$ in the patient and control groups 
While there were no significant difference between patient and control groups for TAS levels, which were $1.39+0.81$ and $1.32+0.20(\mathrm{p}=0.721)$, respectively, the levels of TOS and the OSI were detected significantly higher in the patient group(These values were, respectively, $44.52+0.28$, $10.02+0.97 \quad(\mathrm{p} \leq 0.01) \quad ; \quad 3.92+6.17, \quad 0.78+0.44,($ $\mathrm{p} \leq 0.01$ ) (Table 2).

In the patient group, while there was a strong negative correlation between the serum apelin-36 levels and the HARS scores $(\mathrm{r}=-0.737, \mathrm{p}=0.000)$, correlation was not found between TAS and apelin $36(r=-0.126, p=0.233)$. TOS and OSI were negatively correlated with apelin $36(\mathrm{r}=-0.443, \mathrm{p}=0.026$; $\mathrm{r}=-$ $0.374, \mathrm{p}=0.043$, respectively) (Table 3 ).

\section{Discussion}

There have been recent studies on investigating the role of oxidative stress and biomarkers in the etiopathogenesis of GAD. However, the precise causes have not been identified yet.

Therefore, we investigated the relationship between apelin-36 levels, oxidative stress and GAD patients in this study.The serum apelin-36 levels of the patients with GAD were compared with the healthy controls among with their respective HARS, TAS, TOS, and OSI scores.

The role of oxidative stress biomarkers in the etiopathogenesis of psychiatric disorders including GAD has been studied in previous studies. Ercan et al, showed that prolidase levels were elevated in GAD patients (25). It has been showed that molecules like prolidase, malondialdehyde, superoxide dismutase, and nitric oxide play role in the development of psychiatric conditions including schizophrenia, depression, obsessive-compulsive disorder, and adult attention-deficit hyperactivity disorder (26-30).

In this study, serum apelin-36 levels were found significantly lower in the patient group than the control group. Moreover, there was also a strong negative correlation between the serum apelin-36 levels and the HARS scores in the patient group. In some studies, GAD has been shown to be associated with a proinflammatory response. The detection of inflammatory cytokines such as IL-1, IL-6 and TNF$\alpha$ in the hippocampus and amygdala indicates that they may play a role in etiopathogenesis $(2,4)$. Apelin has been shown to inhibit the secretion of these cytokines in the same regions of the brain (7-10). In addition to its anti-pro-inflammatory cytokine effect in the brain, apelin was shown to have a neuroprotective effect by preventing neuronal apoptosis observed in patients with $\operatorname{GAD}(3,8)$.
Previous studies evaluating the relationship between apelin, and psychiatric diseases have focused on the sub-unit of apelin-13 (12-18). In these studies, it was reported that apelin-13 level was low in autism and may play a role in the etiopathogenesis of ADHD (13). In addition, apelin administered to mice has been shown to have anti-depressive and anxiolytic effects (14-18). No study has been found in the literature investigating the role of apelin 36 in the etiopathogenesis of generalized anxiety disorder.

It is probably that apelin prevents apoptotic processes in neuronal cells by inhibiting the release of anxiogenic and inflammatory cytokines. Therefore, apelin may have a role in the etiopathogenesis of generalized anxiety disorder. Therefore, further studies are needed to clarify the role and the relation of Apelin in GAD.

The envelope phospholipid integrity of neurones in the cortical regions, including amygdala and the hippocampus, is impaired by the accumulation of free radicals and / or the lack of anti-oxidants. These alterations affect the density and functions of catecholamine receptors such as GABA and serotonin / dopamine / noradrenaline which play a role in the pathophysiology of GAD (31-32). In addition, it can also mask serotonin binding sites of the receptor, and thus may play a role in the etiopathogenesis (33).

In this study, even though TAS was similar in both groups, TOS and OSI values were significantly higher in patients. Cenk et al, also reported that it was found similar TAS levels in GAD patients and controls. However, Emhan et al, reported that higher levels of TAS was found in GAD patients than controls $(25,34)$. In both studies, TOS and OSI were like ours. According to these results, inflammatory cases characterized by free radical accumulation may play a role in the etiology of GAD.

Apelin and its receptors are commonly found in the structures organizing stress response such as hypothalamus, hippocampus, and amygdala (8-10). Apelin inhibits the inflammatory response by decreasing release of cytokines such as IL-1, IL-6 and TNF- $\alpha$ in these brain regions. Some studies have shown that apelinergic system protects neurons by preventing apoptosis and improves behavioral performance (7-10).

Free radicals cause apoptosis in the hypothalamus, hippocampus, and amygdala regions of the CNS by disrupting synaptic plasticity, stimulating / increasing inflammatory cytokine release, and triggering preapoptotic signaling (19).

This study also demonstrated a negative correlation between TOS and OSI and apelin-36. However, no correlation was found between TAS and 
apelin-36. Despite it has a potent bioactivity in neuronal protection, the role of apelin-36 in the etiopathogenesis of GAD has not been investigated so far. The small number of participants in the study and the fact that inflammatory cytokine and apelin-36 levels were not measured before and after treatment are the limiting factors.

\section{Conclusion}

According to the results of this study it could be taken into consideration that free radical accumulation and low apelin-36 may play a role in the etiopathogenesis of GAD.

Ethics Committee Approval: This study was conducted with approval from our faculty of medicine ethics committee (Report no: 2016/96)

Peer-review: Externally peer-reviewed.

\section{Author Contributions:}

Concept: IB, USS, BB, Design: MP, NA, OFD, Literature search: IB, MP, BB Data Collection and Processing: USS, NA, OFD, Analysis or Interpretation: $\mathrm{MP}, \mathrm{IB}, \mathrm{USS}$, Writing: $\mathrm{IB}, \mathrm{BB}, \mathrm{ZAY}$ Conflict of Interest: No conflict of interest was declared by the authors.

Financial Disclosure: The authors declared that this study hasn't received no financial support.

\section{References}

1. Stein MB. Public Health Perspectives on Generalized Anxiety Disorders. J Clin Psychiatry 2004;65: 3-7.

2. Pitsavos C, Panagiotakos D, Papageorgiou C, Tsetsekou E, Soldatos C, Stefanadis C. Anxiety in relation to inflammation and coagulation markers, among healthy adults: the ATTICA study. Atherosclerosis 2006;185(2):320-6.

3. Ding L, Zhang C, Masood A, Jianxin Li, Jiao Sun et al. Protective effects of phosphodiesterase 2 inhibitor on depression and anxiety like behaviors: Involvement of antioxidant and anti-apoptotic mechanisms. Behav Brain Res 2014;268:150-8.

4. Mcgaugh J, Mclntyre C, Power A. Amygdala modulation of memory consolidation: İnteraction with other brain systems. Neurobiol Learn Mem 2002;78(3):539-52.

5. Tatemoto K, Hosoya M, Habata Y, Fujii R, Kakegawa et al. Isolation and characterization of a novel endogenous peptide ligand for the human APJ receptor. Biochem Biophsy Res Commun1998;251(2):471-6.
6. Hosoya M, Kawamata Y, Fukusumi S, Fujii R, Habata $\mathrm{Y}$ et al. Molecular and functional characteristics of APJ: Tissue distribution of mRNA and interaction with the endogenous ligand apelin. J Biol Chem 2000;275(28):2106121067.

7. Sakamoto K, Murakami Y, Sawada S, Ushikubo $\mathrm{H}$, Mori A et al. Apelin-36 is protective against Nmethyl-D-aspartic-acid-induced retinal ganglion cell death in the mice. Eur $\mathrm{J}$ Pharmacol 2016;791:213-220.

8. O’Donnell LA, Agrawal A, Sabnekar P, Dichter MA, Lynch KD. Apelin, an endogenous neuronal peptide, protects hippocampal neurons against excitotoxic injury. J Neurochem 2007;102(6):1905-1917.

9. Japp AG, Cruden NL, Amer DAB, Vivienne KY $\mathrm{Li}$, Goudie EB et al. Vascular Effects of Apelin In Vivo in Man. J Am Coll Cardiol 2008;52(11):908-913.

10.Gu Q, Zhai L, Feng X, Chen J, Miao Z et al. Apelin-36, a potent peptide, protects against ischemic brain injury by activating the PI3K/Akt pathway. Neurochem Int 2013;63: 535-40.

11.Kutlay $\mathrm{O}$. The impact of apelin-36 on isolated rat hearts as a member of apelin family Bratisl Med $\mathbf{J}$ 2018; 119(10): 625-9.

12.Duman H, Bahceci İ, Hamur H, Demirelli S, Dilek AR et al. The Relationship between Serum Apelin Level $\mathrm{s}$ and the Severity of Calcific Aortic Stenosis. Acta Cardiol Sin 2018; 34 (3): 259-66.

13. Bilgiç A, Toker A, Uysal S. Exploratory study to evaluate plasma vasopressin and apelin-13 levels in children with attention-deficit hyperactivity disorder. Psychiatry Clin Neurosci 2016;70(10):442-7.

14. Boso M, Emanuele E, Politi P, Pace A, Arra M et al. Reduced Plasma Apelin Levels in Patients with Autistic Spectrum Disorder. Arch Med Res 2007;38(1):70-4.

15.Li E, Deng H, Wang B, Fu W, You Y, Tian S. Apelin-13 exerts antidepressant-like and recognition memory improving activities in stressed rats. Eur neuropsychopharmacol 2016;26(3):420-30.

16.Lv Sy, Qin YJ, Wang HT, Xu N, Yang YJ Chen Q. Centrally administered apelin-13 induces depression-like behavior in mice. Brain Res Bull 2012;88(6):574-80.

17. Telegdy G, Adamik A, Jászberényi $M$. Involvement of neurotransmitters in the action of apelin-13 on passive avoidance learning in mice. Peptides 2013;39(1):171-174. 
18.Xin Q, Cheng B, Pan Y, Liu Haiging, Yang Chunging et al. Neuroprotective effects of apelin13 on experimental ischemic stroke through suppression of inflammation. Peptides 2015;63:55-62.

19.Kokacya MH, Bahceci B, Bahceci I, Dilek AR, Dokuyucu R. Prolidase activity and oxidative stress in patients with major depressive disorder. Psychiatr Danub 2014;26:314-8.

20.Erel O. A new automated colorimetric method for measuring total oxidant status. Clin Biochem 2005; 38 (12): 1103-1111.

21.First MB, Spitzer RL, Gibbon M WJ. Structured Clinical Interview for DSM-IV Clinical Version (SCID-I/CV). Washington DC: American Psychiatric Press, 1997: 25-35.

22. Corapcioglu A, Aydemir Ö, Yıldız M, Esen Danaci A, Koroglu E. DSM-IV Eksen-I Structured Clinical Interview for Disorders. Ankara: Physicians Publishing Union,1999: 1-7.

23. Hamilton M. The assessment of anxiety states by rating. Br J Med Psychol 1959;32:50-5.

24. Yazici MK, Demir B, Tanriverdi N, Karaagaoglu E, Yolac P. Hamilton Anxiety Rating Scale: Interrater reliability and validity study January 1998. Turk Psikiyatri Derg 1998;9:114-7.

25.Ercan AC, Bahceci B, Polat S, Cenker OC, Bahceci I et al. Oxidative status and prolidase activities in generalized anxiety disorder. Asian J Psychiatr. 2017;25: 118-22.

26. Bahceci B, Bagcioglu E, Kokacya MH, Dilek AR, Bahceci I, Selek S. Prolidase activity and oxidative stress in patients with schizophrenia: A preliminary study. J Pak Med Assoc 2015;65(2): 131-35.

27.Bahceci B, Kokacya MH, Copoglu US, Copoglu $\mathrm{S}$, Bahceci I et al. Elevated nucleosome level and oxidative stress in schizophrenia patients. Bratisl Med J 2015;116(10): 587-90.

28. Akyol O, Zoroglu SS, Armutcu F, Sahin S, Gurel A. Nitric oxide as a physiopathological factor in neuropsychiatric disorders. In vivo 2004; 18 : 377 90.

29. Bulut M, Selek S, Gergerlioglu HS, Savas HA, Yilmaz HR et al. Malondialdehyde levels in adult attention-deficit hyperactivity disorder. J Psychiatry Neurosci 2007; 32 (6): 435-438.

30.Ozdemir E, Cetinkaya S, Ersan S, Kucukosman S, Ersan EE. Serum selenium and plasma malondialdehyde levels and antioxidant enzyme activities in patients with obsessive-compulsive disorder. Prog Neuropsycho pharmacol Biol Psychiatry 2009; 33: 62-5.

31.Graham DG. On the origin and significance of neuromelanin. Arch Pathol Lab Med 1979; 103: 359-62.

32.Britt SG, Chiu VW, Redpath G, Vandenberg SR. Elimination of ascorbic acid-induced membrane lipid peroxidation and serotonin receptor loss by Trolox-C, a water-soluble analogue of vitamin E. J Recept Res 1992; 12: 181-200.

33. Nikolaus S, Antke C, Beu M, Müller HW. Cortical GABA, striatal dopamine and midbrain serotonin as the key players in compulsive and anxiety disorders results from in vivo imaging studies. Rev Neurosci2010; 21 (2): 119-39.

34.Emhan A, Selek S, Bayazit H, Karababac IF, Katic M, Aksoy N. Evaluation of oxidative and antioxidative parameters in generalized anxiety disorder. Psychiatry Res 2015; 230: 806-810. 